\title{
Neuropathology of the Acquired Immune Deficiency Syndrome (AIDS): Report of 39 Autopsies from Vancouver, British Columbia
}

\author{
Marcia E. Cornford, Janet K. Holden, Michael C. Boyd, Kenneth Berry and Harry V. Vinters
}

\begin{abstract}
Neuropathological findings from 39 acquired immune deficiency syndrome (AIDS) autopsies of primarily neurologically symptomatic patients and 7 brain biopsies from AIDS patients performed at St. Paul's Hospital, Vancouver, British Columbia are reported. Autopsy findings included human immunodeficiency virus-1 (HIV)-type multinucleated giant cell (MNGC)-associated encephalitis seen in 17 patients, toxoplasmosis in 7 patients, and cytomegalovirus encephalitis and/or microglial nodule-associated nuclear inclusions in brain parenchyma in 9 patients. Central nervous system lymphoma was identified in 11 autopsy patients and in 4 of 7 brain biopsies. Infectious processes including HIV encephalitis were seen in 10 of 11 autopsied patients with lymphoproliferative lesions in the brain parenchyma, while $40 \%$ of patients without lymphoma had HIV-type MNGC or opportunistic infections. CNS lymphoma was not significantly increased in incidence in patients with a clinical history of zidovudine treatment, but increased duration of survival after the diagnosis of AIDS was associated with increased incidence of lymphoma in both untreated and zidovudine-treated patients. Patients displaying HIV MNGC within microglial nodules had a shorter mean duration of survival after diagnosis of AIDS than those patients with HIV encephalitis with dispersed MNGC, white matter vacuolation, and gliosis.

RÉSUMÉ: Neuropathologie du syndrome d'immuno-déficience acquise: compte rendu de 39 autopsies de Vancouver, Colombie Britannique. Nous rapportons les constatations neuropathologiques sur 39 cas de syndrome d'immuno-déficience acquise (SIDA), ayant subi une autopsie, chez des patients avec des manifestations neurologiques prédominantes et 7 biopsies du cerveau chez des sidéens, effectuées à l'hôpital St-Paul de Vancouver, Colombie Britannique. A l'autopsie, on a observé une encéphalite associée à des cellules géantes multinuclées (CGMN) de type HIV (virus de l'immuno-déficience humaine) chez 17 patients, une toxoplasmose chez 7 patients et une encéphalite à cytomégalovirus et/ou des inclusions nucléaires associées à des nodules microgliaux dans le parenchyme cérébral chez 9 patients. Un lymphome du système nerveux central a été identifié à l'autopsie chez 11 patients et dans 4 des 7 autopsies du cerveau. Un processus infectieux incluant une encéphalite à HIV a été observée à l'autopsie de 10 patients sur 11 ayant des lésions lympho-prolifératives du parenchyme cérébral, alors que $40 \%$ des patients sans lymphome avaient une infection à CGMN de type HIV ou une infection opportuniste. L'incidence du lymphome du SNC n'était pas augmentée de façon significative chez les patients avec une histoire clinique de traitement à la zidovudine, mais une survie plus longue après un diagnostic de SIDA était associée à une incidence accrue de lymphome, tant chez les patients non traités que ceux traités à la zidovudine. Les patients ayant des CGMN de type HIV dans des nodules microgliaux avaient une survie moyenne plus courte après le diagnostic de SIDA que les patient présentant une encéphalite HIV avec CGMN dispersées, vacuolisation de la substance blanche et gliose.
\end{abstract}

Can. J. Neurol. Sci. 1992: 19:442-452

Since the beginning of the AIDS epidemic, a number of autopsy studies have documented the incidence of the various neuropathological lesions found in AIDS patients from different geographic regions, including Southern California, ${ }^{1.2}$ Northern California; ${ }^{3}$ New York; ${ }^{4,5}$ Boston; ${ }^{6.7}$ Miami, Florida; ${ }^{8}$ Britain; ${ }^{9.10}$ France; ${ }^{11.12}$ Switzerland; ${ }^{13,14}$ Italy; ${ }^{15}$ Austria; ${ }^{16}$ Germany; ${ }^{17}$
Brazil; ${ }^{18}$ and Japan. ${ }^{19}$ Analysis of these reports reveals possible regional differences in the incidence of the various neuropathological lesions, as well as documenting important contrasts between different "at risk" populations such as women, ${ }^{4}$ children, ${ }^{4.20}$ hemophiliacs, ${ }^{9.10}$ intravenous drug users of both sexes, ${ }^{4.16}$ and the more frequently studied populations of male

From the Department of Pathology (M.E.C., H.V.V.), and Brain Research Institute (H.V.V.), UCLA Center for Health Sciences, Los Angeles; Departments of Pathology (J.K.H.) and Surgery (M.C.B.), St. Paul's Hospital, Vancouver; Department of Pathology (K.B.), Vancouver General Hospital, Vancouver

Received February 21, 1992. Accepted in final form June 25, 1992

Reprint requests to: Dr. Marcia E. Cornford, Department of Pathology, UCLA Center for Health Sciences, 10833 Le Conte Ave., Los Angeles, CA $90024-1732$ 
homosexuals. The regional differences include variable incidence of cerebral toxoplasmosis, ranging from $30 \%$ in Miami, Fla., ${ }^{8}$ to 6 and $7 \%$ in series from Southern California; ${ }^{1.2}$ central nervous system lymphoma, ranging from $2.5 \%$ in New York ${ }^{2 !}$ to $7.4 \%$ in the most recent survey from San Francisco; ${ }^{3}$ and HIVassociated encephalitis (with characteristic multinucleated giant cells) ${ }^{22.23}$ ranging from $19 \%$ in Switzerland ${ }^{13}$ to $30 \%$ in a report from Austria. ${ }^{16}$ These reports provide information on the likely diagnostic possibilities to be considered in the evaluation of AIDS patients with neurological symptoms in any venue, as well as helping to define the effects of HIV-1 viral infection and the opportunistic infectious organisms or neoplasms causing disease in the immunodeficient individual.

The present study is derived from a series of autopsies and brain biopsies performed at St. Paul's Hospital, Vancouver, British Columbia, from 1984 to 1991. This hospital serves an urban population in which HIV infection risk factors of homosexuality and intravenous drug usage are prevalent. The incidence of central nervous system lymphoma, infectious manifestations of opportunistic organisms, and the patterns of HIV-1 infection ${ }^{22}$ are documented for 39 autopsies and 7 brain biopsies from patients who were not subsequently autopsied.

\section{Materials and Methods}

Post mortem gross examinations and sampling for light microscopic examination were performed on HIV-infected patients who died at St. Paul's Hospital, Vancouver between May 1984 and March 1990, with AIDS-defining illnesses by 1987 Centers for Disease Control (USA) criteria, ${ }^{24}$ and/or HIV-1 seropositivity after the introduction of the serum assay in 1985. The patients were principally selected for autopsy on the basis of need for a definitive diagnosis of neurological disorders. Fixation of brains in $10 \%$ neutral buffered formalin for 2 weeks was followed by coronal sectioning, gross examination and photographic documentation of visible lesions, and systematic sampling of the entire brain. Light microscopic sections from frontal cortex, basal ganglia, thalamus, parietal cortex, hippocampus, midbrain, pons, medulla, cerebellum and any grossly visible lesions were obtained from most patients. Paraffin-embedded material was stained with hematoxylin and eosin, with appropriate special stains applied as necessary (Periodic acid-Schiff, Gram, Giemsa, Fite acid-fast bacilli, and Gomori methenamine silver). Neuropathological changes for each patient were recorded with respect to distribution, severity and associated lesions, before details of the clinical history were ascertained from hospital medical records to determine length of survival with the diagnosis of AIDS, therapy with zidovudine, and other diagnoses of infectious organisms or neoplasms.

Biopsy specimens were typically obtained at open biopsy from patients with significant radiographically detectable lesions, or with rapidly progressive dementia and encephalitic symptoms. Such material was also processed in paraffin with routine hematoxylin and eosin staining of step sections through the specimen, and appropriate special stains for infectious organisms performed as needed. While some autopsied patients also had surgical biopsies, each is counted only once in statistical evaluation. Statistical comparisons of the incidence of the most common AIDS-related neuropathologic findings in patients who had been treated with zidovudine and those who had not been treated were performed with Chi square analysis of the significance of the differences of the number of patients in each group.

All patients included in this study were Caucasian, with the exception of one male Oriental, and three of 46 were female. Known risk factors were assessed to be homosexuality in 40 patients, intravenous drug usage in 2 patients, blood transfusion in 2 patients, and heterosexual exposure in 2 patients. The mean age of the autopsied patients studied was 38.0 years, with a range of 20 to 67 years. Eight autopsied and two biopsied patients had received zidovudine therapy for at least one month. The population of AIDS patients from which the subjects were selected included 390 deaths of HIV infected persons at St: Paul's Hospital during the time period of the study. Patients studied were typically autopsied to establish a definitive cause of neurological symptoms, or to examine the cause of unexpected death in six patients. Therefore, this survey is not necessarily indicative of the incidence of neuropathologic abnormalities in a majority of AIDS patients in this region, but is fairly representative of those patients dying with a significant neurological syndrome. The distribution of numbers of patients autopsied in each year of the study is as follows: 3 in 1984, 6 in 1985, 7 in 1986, I in 1987,8 in 1988,7 in 1989 , and 7 in 1990. Surgical biopsies were obtained in 1987, ${ }^{3} 1988,,^{\prime} 1989,{ }^{\prime} 1990^{1}$ and 1991.'

\section{RESULTS}

Light microscopic observations: Table I presents a summary of the principal neuropathological lesions found in the 39 autopsied patients examined, their demographic identification, and other diagnoses. Patients who had received zidovudine treatment are denoted $\left({ }^{*}\right)$. The four most frequently identified conditions were HIV encephalitis ( 17 patients), central nervous system lymphoma (11 patients), toxoplasmosis (7 patients), and cytomegalovirus infection ( 9 patients). Table 2 summarizes data from 7 surgical biopsies of AIDS patients who were not autopsied. Table 3 presents a summary of the several categories of neurological disease and the interrelationships of infectious organisms and lymphoma in the 39 autopsied patients.

HIV encephalitis: Recent reports have emphasized the presence of different patterns of apparently progressive destructive lesions associated with sites of infection by HIV-1 in brain parenchyma. ${ }^{22.23}$ One of the patterns described (HIV encephalitis) consists of focal grey and white matter microglial nodule (MGN) inflammatory lesions associated with one to several multinucleated giant cells (MNGC) containing many small vesicular nuclei resembling those of microglial cells, with the surrounding parenchyma essentially unchanged. The other pattern (HIV leucoencephalopathy) is described as scattered MNGC of HIV type within vacuolated white matter, with pigmented debris adjacent to capillary profiles, and astrocytic gliosis. Irregular regional involvement of the brain is characteristic and overlap of the patterns is occasionally found.

In the current study, five patients displayed foci of several MNGC associated with discrete MGN only, usually in several regions of the brain. Nine patients had regional involvement of diffusely distributed MNGC, appreciable gliosis and white matter vacuolation in surrounding parenchyma, and no discrete MGN. Both patterns were found in different regions of the brains, in three patients. The mean duration of survival after the diagnosis of AIDS for patients with the microglial nodule pattern 


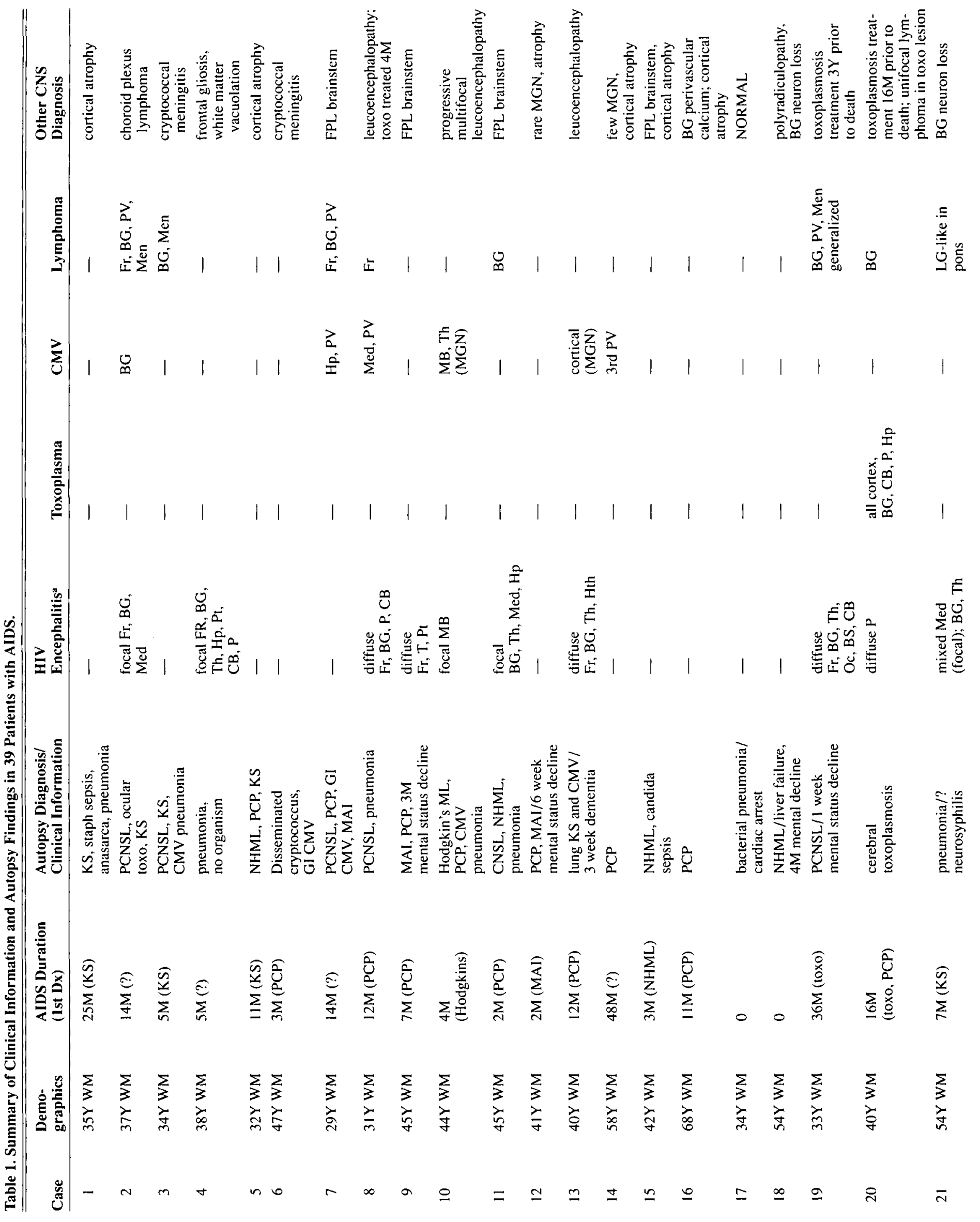




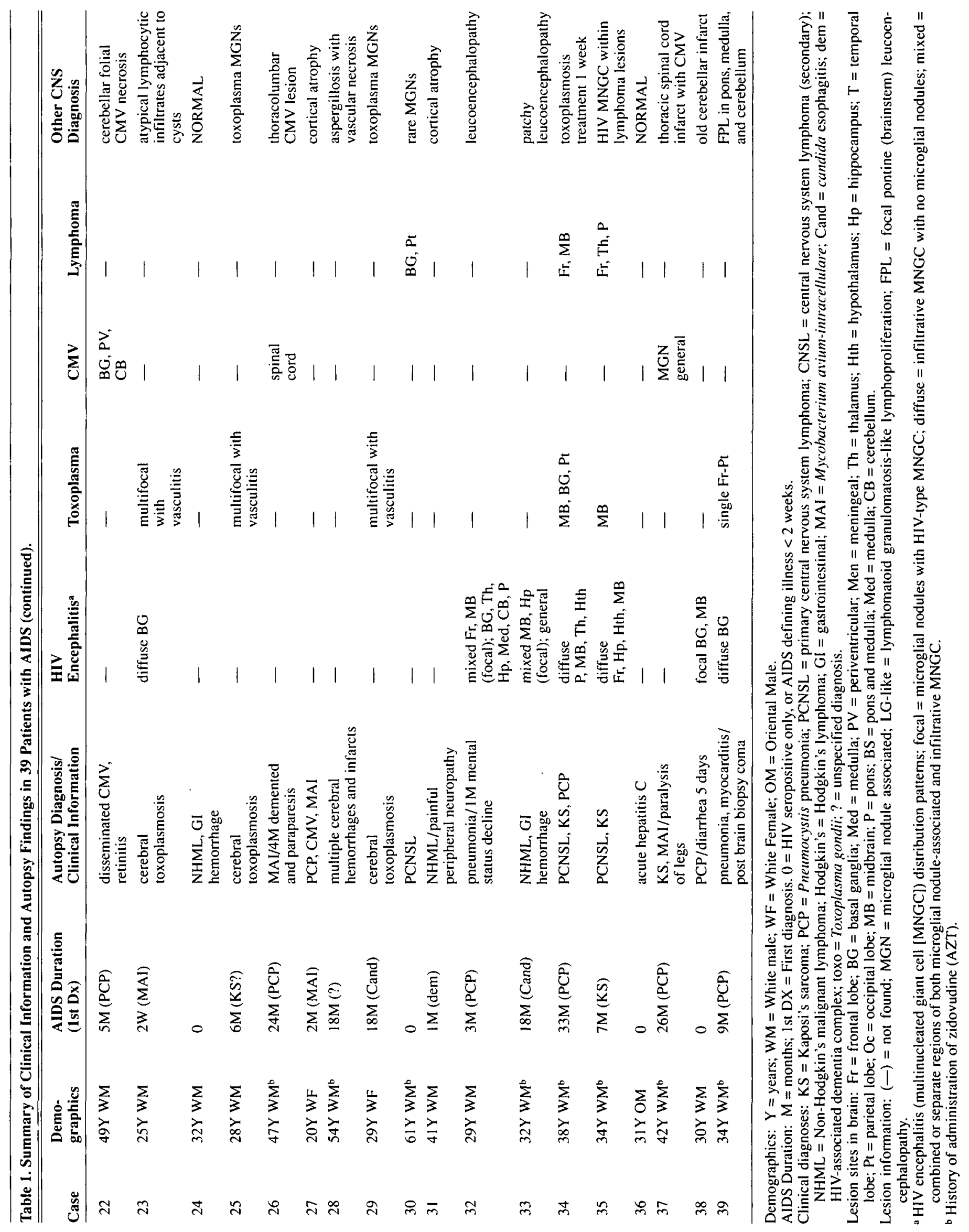


Table 2. Summary of Neuropathological Findings in 7 Brain Biopsies of AIDS Patients.

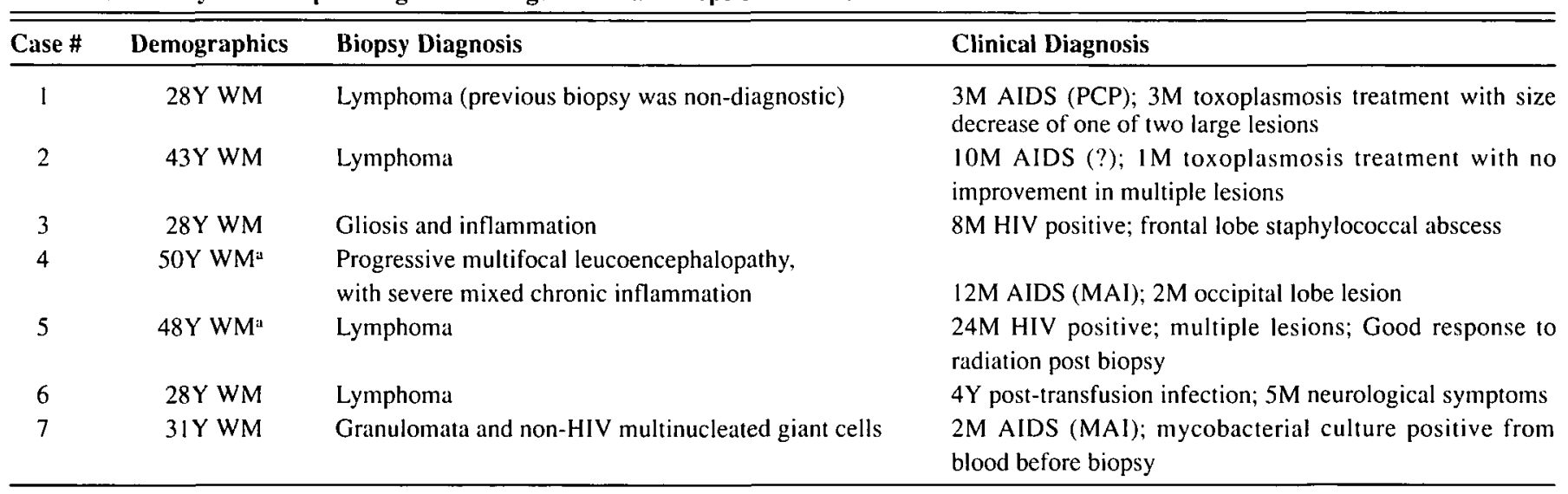

Demographics: $Y=$ years; $W M=$ White Male; $W F=$ White Female

Clinical diagnosis: $\mathrm{M}=$ months; $\mathrm{PCP}=$ Pneumocystis carinii pneumonia; $\mathrm{MAI}=$ Mycobacterium avium/intracellulare

$a=$ History of administration of zidovudine $(A Z T)$.

Table 3. Distribution of Neuropathologic Lesions in 39 Autopsy Patients with AIDS (St. Paul's Hospital, Vancouver)

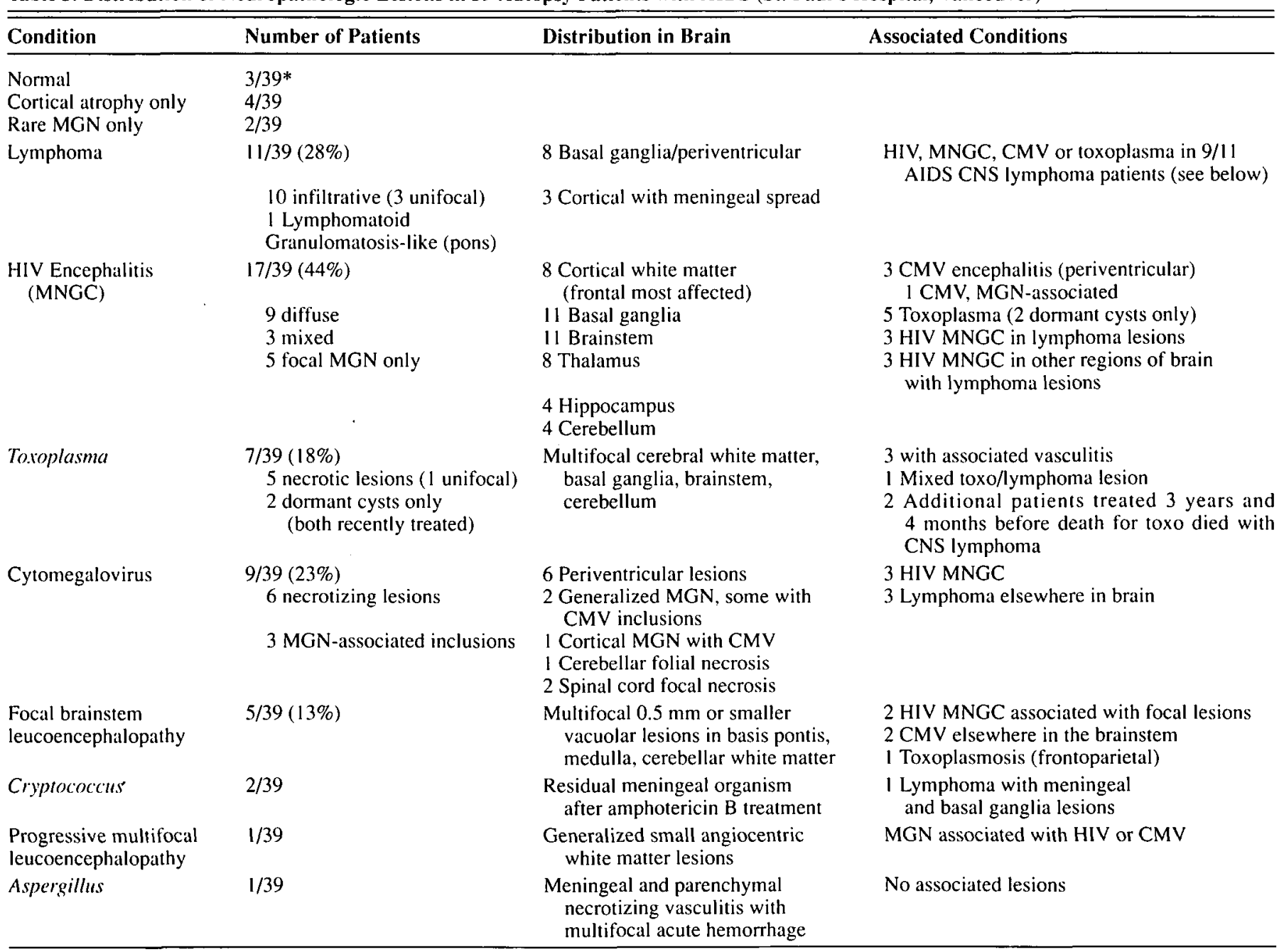

MNGC: multinucleated giant cells of HIV-type

MGN: microglial (focal aggregation) nodules

* All three patients with normal-appearing brains at autopsy were HIV-positive individuals who died after a brief illness with no AIDS-defining criteria. 
was 5 months (range 0 to 14 months); for patients with the more destructive-appearing diffuse infiltration of MNGC pattern, mean survival was 14.7 months ( 2 weeks to 36 months); and for the overlapping pattern, mean survival was 9.3 months ( 3 to 18 months). On this basis, it is suggested that MGN-associated HIV lesions may appear at an earlier stage of brain infection by HIV than the diffuse infiltrative distribution of HIV-type MNGC, and possibly may precede the latter pattern.

The sites most often affected by MNGC-associated lesions were frontal white matter, basal ganglia, and thalamus. In all patients with HIV-type MNGCs, at least some of the brain examined appeared to lack these cells, and had a more normal appearance than parenchyma containing either of the patterns of HIV infection mentioned above. Brains with MNGC associated with discrete microglial aggregations were often found to have many other capillary-centered MGN without MNGCs. The diffuse distribution of MNGC, principally in white matter tracts and basal ganglia, was associated with obvious parenchymal rarefaction, prominent astrocytosis, and coarse vacuolation. The diffuse pattern tended to contain more capillary-centered brownpigmented debris and generally showed more advanced degenerative changes than the focal HIV MNGC-associated microglial nodule pattern. These changes included degeneration of scattered neurons in the basal ganglia, and capillary basement membrane calcification in the putamen of five patients.

HIV encephalitis, defined to be present when MNGC were found, was present in 4 of 8 zidovudine-treated patients, and in 13 of 31 patients with no zidovudine therapy. Chi square analysis indicates that this difference is not significant $(p>0.2)$. The interval since discontinuation of zidovudine was not considered in this study.

Lymphoma: Eleven of the 39 autopsied patients studied had central nervous system lymphoproliferative lesions (Figure 1); only one of these patients had systemic lymphoma. Seven patients had multifocal infiltrative lymphoma, with similar cellular morphology in all foci, while three patients displayed unifocal infiltrative lymphoma, and one patient had a unifocal lymphomatoid

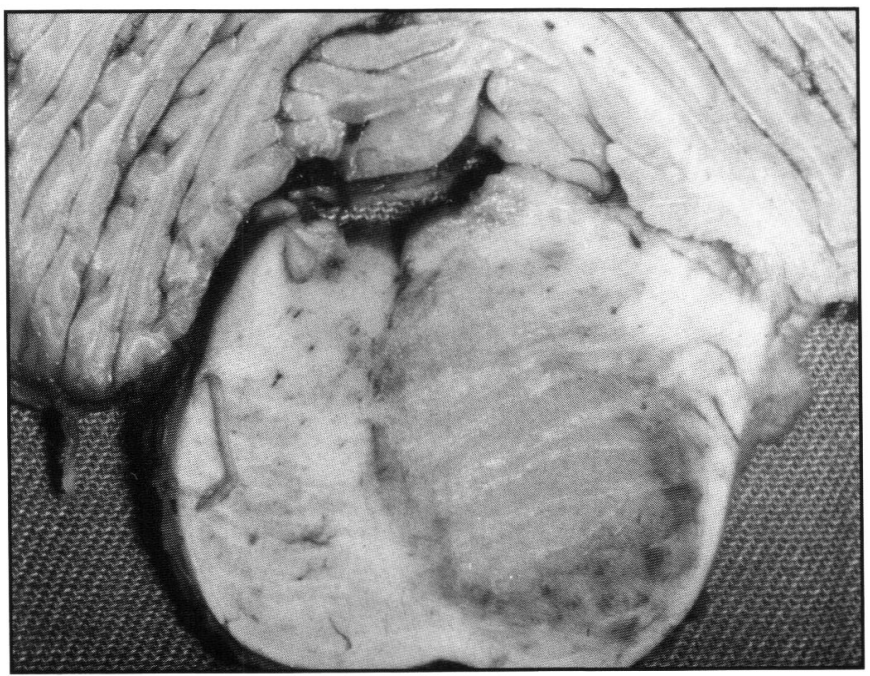

A granulomatosis-like angiocentric pattern ${ }^{25}$ of markedly atypical lymphocytes (Case 21). The regions of distribution of the multifocal lesions indicated meningeal spread in three patients, periventricular spread in eight patients, and evidence of both in two patients.

Table 3 indicates the distribution of lesions within the brain for all patients, and the association of lymphoproliferative lesions with opportunistic infection by Toroplasma gondii and cytomegalovirus, as well as morphologically distinctive HIV-I lesions (MNGCs). Nine of the eleven patients with lymphoproliferative lesions also had at least one of these three infectious organisms present $(82 \%)$, in the relatively modest parenchymal sampling used. These three types of infectious lesions were found in 12 of 28 patients (43\%) with no lymphoproliferative lesions in this series. An additional patient with treated cryptococcal meningitis had extensive infiltration of lymphoma within meninges and adjacent brain parenchyma.

Lymphoma lesions in five patients contained HIV-type MNGC, or had them at the periphery of lesions (Figure 1). Association of an atypical lymphocytic proliferation with a necrotic focus of toxoplasmosis was seen in one patient (Figure 2). The lymphoma lesions were morphologically typical of B-cell lesions in all cases, with four patients showing a pattern consistent with small, non-cleaved cell type, two consistent with pleomorphic large cell type, and five typical of immunoblastic lymphoma.

Zidovudine-treated patients accounted for three of the cases of primary central nervous system lymphoma (PCNSL), from a total of eight treated autopsied patients included in the study. The remaining 31 untreated patients included seven with PCNSL, and one with central nervous system involvement by systemic lymphoma. Systemic lymphoma had been previously diagnosed prior to death and treated, or was evident at autopsy, in six patients not treated with zidovudine (five non-Hodgkin's and one Hodgkin's lymphoma), and one zidovudine-treated patient. Chi square analysis of the significance of the difference in incidence of PCNSL $(p>0.1)$, and in the incidence of all lymphoma $(p>0.1)$ between zidovudine-treated and untreated patient groups showed that the differences were not significant. The mean survival after the diagnosis of AIDS in all zidovudine-

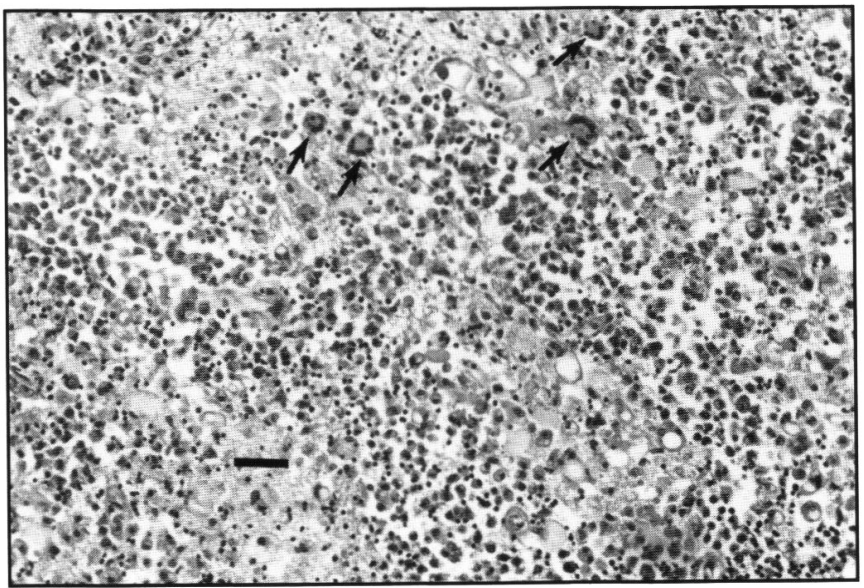

B

Figure IA - Pontine lymphoma (Case 35). B. Micrograph of a portion of the same lesion showing HIV-type multinucleated giant cells (arrows) within an infiltrative, partially necrotic lymphoma lesion. Hematoxylin and eosin. Magnification bar = 50 micrometers. 


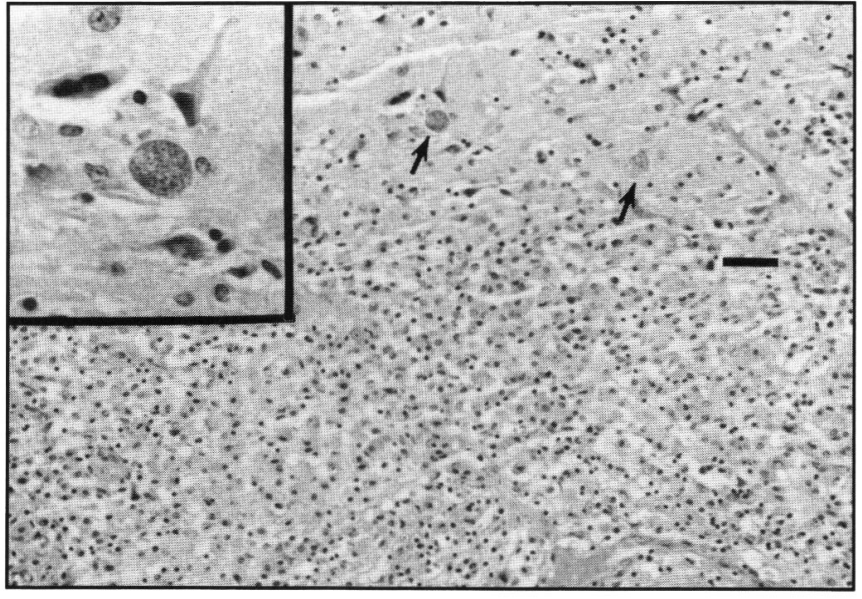

Figure 2 - Toxoplasma cysts (arrows) at the border of an atypical lymphocytic infiltrate in a necrotic lesion, in a patient with multifocal necrotizing toxoplasmosis (Case 23). Inset: Toxoplasma cyst. Hematoxylin and eosin. Magnification bar = 50 micrometers.

Ireated persons was 18.0 months (range 0 months to 33 months), while the mean survival of all non-treated patients was 6.9 months (range 0 months to 48 months). The mean survival after the diagnosis of AIDS for patients without lymphoma was 8.3 months, while zidovudine-treated patients with PCNSL survived an average of 14.1 months (range 0 to 33 months) and untreated patients, 13.3 months (range 2 to 33 months). The mean survival of patients with either PCNSL or systemic lymphoma was also longer than the mean survival of patients without lymphoma: 14.5 months (range 0 to 33 months) for zidovudine-treated patients, and 9.1 months (range 0 to 36 months) for untreated patients. Survival after the appearance of neurological symptoms attributable to intracranial mass lesions of central nervous system lymphoma was less than 4 months for all of the patients studied. The incidence of lymphoma therefore appears to increase with increased duration of survival with the diagnosis of AIDS in both zidovudine-treated and untreated groups.

Toxoplasmosis: Toxoplasmosis was evident in seven patients, with extracerebral Toxoplasma not found in any of the patients, although ocular infection was demonstrated in one patient without cerebral toxoplasmosis. Severe necrotic toxoplasmosis lesions were deemed to be the cause of death in three patients, and one other patient died of post-biopsy complications. Inflammation of parenchymal vessels (vasculitis) was seen in one or more of the multifocal lesions of the three patients in whom they were the cause of death, with small, petechial hemorrhages found in two. Microglial nodules were observed at the periphery of cyst-containing lesions, and were thought to be Toxoplasma-associated in three patients. Two of the patients with Toxoplasma cysts lacked any associated inflammation or necrosis; both had had recent toxoplasmosis treatment. Three patients with toxoplasmosis at autopsy also had lymphoproliferative lesions, including one with atypical lymphocytic infiltrates around necrotic lesions with numerous Toxoplasma cysts at the periphery (Figure 2). Another patient (Case 20) with a clinical history of cerebral toxoplasmosis had treatment for toxoplasmosis after the recurrence of neurological symptoms for 1 week, and no histological evidence of cysts, but demonstrated infiltrative lymphoma within a large basal ganglia lesion, along with multifocal centrally necrotic, partially calcified lesions typical of toxoplasma infection. Two other patients having no histological evidence of past or present Toxoplasma infection at the time of autopsy had been treated for intracerebral toxoplasmosis 3 years and 4 months prior to death; both expired with primary central nervous system lymphoma.

Cytomegalovirus: Cytomegalovirus (CMV) parenchymal infection, usually involving periventricular areas, was evident in 6 of the 39 autopsy cases studied; three other patients had CMV nuclear inclusions within microglial nodule-associated capillary endothelial cells. Combined CMV/HIV, CMV/Toxoplasma, and $\mathrm{CMV} /$ lymphoma lesions were not identified in any of the patients, although patients with CMV encephalitis also demonstrated HIV MNGC (3 patients) and lymphoma (3 patients) in other regions of the brain. Two patients with extensive infarctlike lesions of spinal cord dorsal columns displayed CMV nuclear inclusions in vascular endothelial cells within these lesions. Both patients had capillary-associated MGN, some with endothelial CMV inclusions, in several regions of their brains. Severe focal CMV infection of cerebellar folia, with inclusions found in Purkinje cells, as well as cells of the molecular and granule cell layers, was found in one patient (Figure 3 ).

Focal pontine (brainstem) leucoencephalopathy: ${ }^{26}$ Five patients showed multiple foci of punctate, intramyelinic vacuolation and axonal spheroid formation in the parenchyma of the brain stem, including the central basis pontis, medulla and central cerebellar white matter (Figure 4). While surrounded by unremarkable parenchyma, these foci displayed little inflammation, and loss of oligodendrocytes in white matter and neurons in grey matter, with adjacent neuroaxonal spheroids. No appreciable calcification was apparent in any of these lesions. Three of the individuals displayed HIV-type MNGCs elsewhere in the brain, and one had these cells within a cerebellar lesion (Figure 4). In addition, two of the five patients who showed several minute foci of white or grey matter vacuolation in the central basis pontis, and central medulla and midbrain, also had periventricular CMV infection of the brain stem. The two patients with extensive infarct-like lesions of thoracic and lumbar spinal cord associated with CMV nuclear inclusions in endothelial cells both had central pontine and focal central medullary focal vacuolations.

Additional neuropathologic findings: Less frequently observed infectious organisms found in the series of autopsies included cryptococcal meningitis in two patients with recent amphotericin B treatment showing a few residual intact organisms. Extensive aspergillosis involving meningeal and parenchymal vessels was found in one patient, who had demonstrated neurological symptoms of headache and acute dementia for a short time prior to death. Extensive parenchymal hemorrhages were seen in the frontal lobe and brainstem of this patient, as a result of transmural acute inflammation of infected blood vessels (Figure 5). Progressive multifocal leukoencephalopathy was the cause of death in one autopsied patient, and was found in a biopsy specimen.

Biopsy Diagnoses: The seven patients with surgical biopsies who did not come to autopsy included four with the biopsy diagnosis of lymphoma, one with progressive multifocal leukoencephalopathy, one with a Staphylococcal abscess, and one patient with meningeal and adjacent parenchymal granulomata 


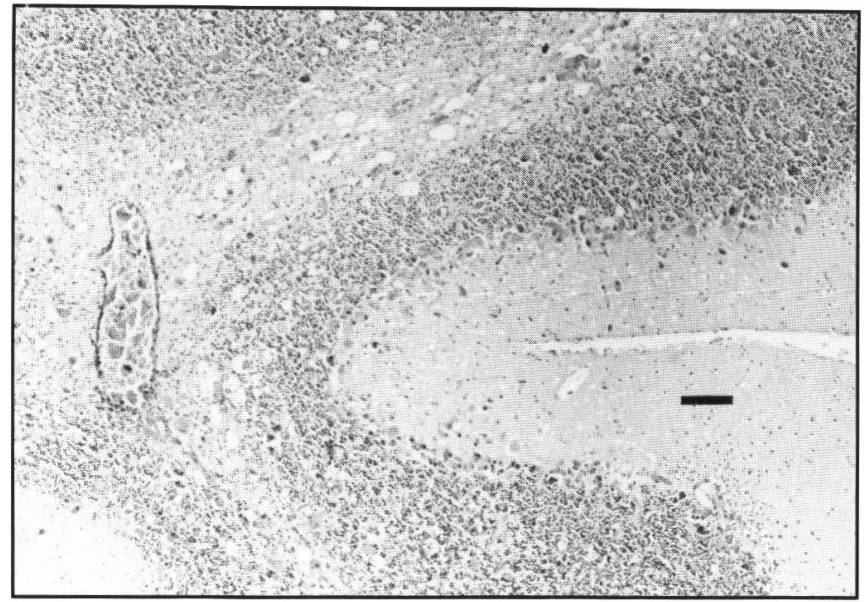

A

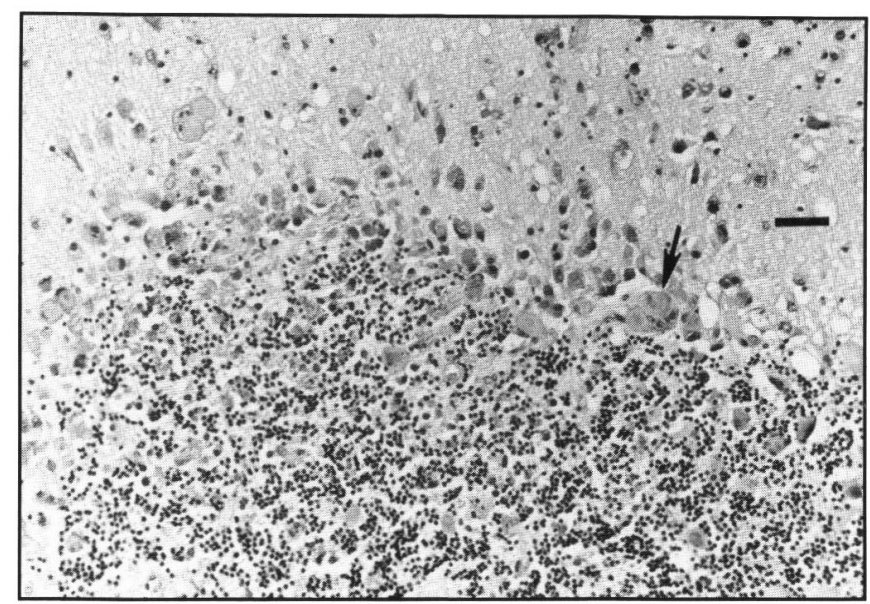

B

Figure 3A. - Focus of cytomegalovirus infection in cerebellar folia (Case 22). B. Higher power micrograph of another portion of the lesion showing cytomegalic cells and nuclear inclusions, including a probable Purkinje cell (arrow). Henatorylin and eosin. Magnification bars $A=200$ micrometers, $B=50$ micrometers.

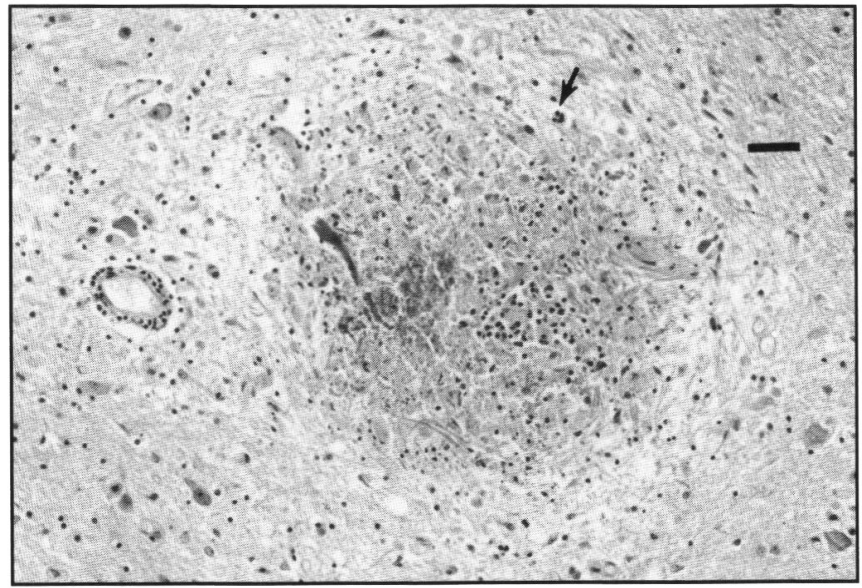

Figure 4-Punctate lesion of focal extra-pontine leucoencephalopathy in the central cerebellar white matter (Case 39). Unremarkable white matter surrounds a focus of necrosis, vacuolation, axonal spheroids, and an HIV-type multinucleated giant cell (arrow). Hematoxylin and eosin. Magnification bar = 50 micrometers.

with no stainable acid-fast bacilli, but a clinical history of positive mycobacterial cultures.

Distribution of Diagnoses: A summary of the distribution of neuropathologic abnormalities identified in the 39 autopsies is presented in Table 3. Multiple infections were found in 10 of the patients. A relatively high percentage of central nervous system lymphoma (28\%) was found, compared to previous series, probably due to the selective inclusion in the series of mostly symptomatic patients. HIV MNGC were present in 17 patients $(44 \%)$. Microglial nodule encephalitis (the subacute encephalitis defined by some authors ${ }^{6}$ ), was found to be associated with HIV in 8 patients, Toxoplasma in three patients, CMV in 3 patients, and two patients with a few microglial nodules did not contain an associated identifiable organism.

\section{Discussion}

The relatively high percentages of neuropathological lesions found in this investigation of all autopsies of AIDS patients at a single Vancouver hospital, representing approximately $10 \%$ of all AIDS patients dying there during this period of time, reflects selection of individuals with significant neurological symptoms. Autopsy bias, discussed by Wiley and Nelson, ${ }^{27}$ tends to accentuate the incidence of serious pathologic abnormalities in any such survey. Careful consideration of the autopsy bias in this study, with a low percentage of cases examined compared to total AIDS patient deaths in the population, should be kept in mind in comparisons with surveys in which higher percentages of all AIDS deaths were autopsied. For example, in the Swiss series $^{1.3}$ in which $73 \%$ (135 of 184 patients) of all AIDS patients were examined for neuropathological abnormalities, and the series from San Francisco studying 94 of 104 consecutive patients, ${ }^{3}$ the percentages of CNS lymphoma were found to be 3.5 and $7.4 \%$, respectively. In the present study, where II CNS lymphomas were demonstrated in 39 patients from a total of 390 AIDS patient deaths, the percentage of the total population affected extrapolates to $2.8 \%$, but it is unlikely that all CNS lymphomas were included in the cases autopsied, despite the bias in the autopsy patients we have studied toward inclusion of those with neurological symptoms. The presence of symptomatic neurological disease in the subjects is not stated in most of the other autopsy series. CMV encephalitis was found in $17 \%$ of the autopsy cases, compared to the percentages found in Los Angeles (16\%), ${ }^{1}$ New York (26\%), ${ }^{5}$ and Switzerland $(7 \%),{ }^{13}$ all in populations with homosexuality as the predominant risk factor. Vasculitis was found to be associated with Toxoplasma infection in three patients, and in one patient with vascular infection by aspergillosis, while no evidence of vascular wall inflammation/destruction was seen in patients with CMV or HIV encephalitis. Two patients with symptomatic destructive lesions of the dorsal columns of the spinal cord were found to show CMV inclusion cells in the lesions. Toxoplasmosis in a fulminant form was evident in 5 of 39 autopsies (13\%). Other neuropathology associated with opportunistic infections was 


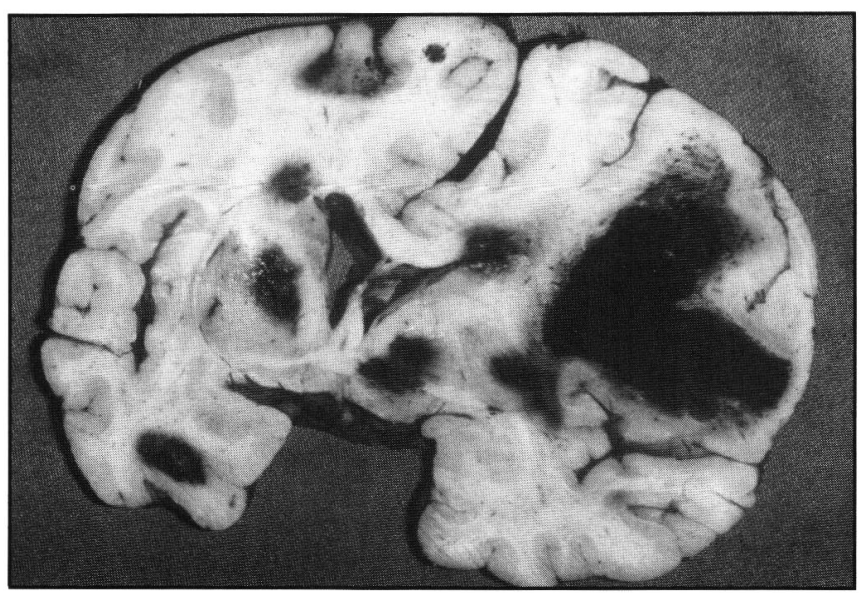

A

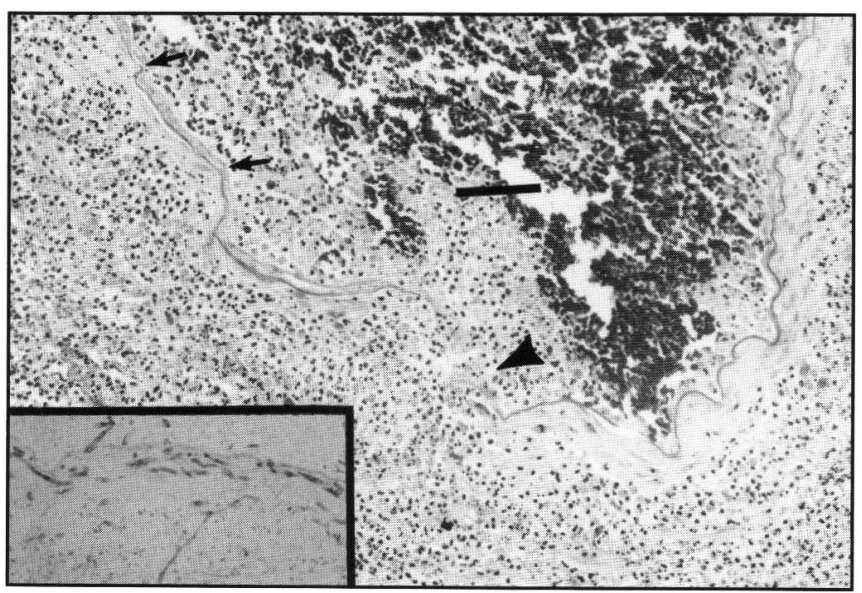

B

Figure 5A. - Coronal section displaying multifocal acute hemorrhages resulting from aspergillosis of many parenchymal blood vessels (Case 28). B. Meningeal vessel of the frontal lobe has extensive acute inflammation and ruptwe of the arterial layers (arrowhead). Small arrow's indicale site of inset. Hematorylin and eosin. Inset. Gomori methanamine-silver stain of the same vessel demonstrates fungal hyphae throughout the wall. Magnification bar $B=200$ micrometers.

also comparable to other series. The lesions of focal pontine (brainstem) leucoencephalopathy, which is usually not considered separately from other manifestations of HIV-related leucoencephalopathy, may be related to the multifocal vacuolar leucoencephalopathy defined primarily in the cerebral hemispheres by Schmidbauer and colleagues ${ }^{28}$ and Budka, ${ }^{22}$ lesions which sometimes contain HIV by immunohistochemistry, or by presence of HIV-type MNGC. Both CMV and HIV infection were evident in the brains of the AIDS patients studied here, but such lesions also occur in the absence of viral infection in patients immunosuppressed for other reasons (26, and unpublished observations by HVV).

HIV encephalitis, defined by the presence of MNGC of HIVtype in the brain parenchyma, rather than the simple presence of microglial nodule encephalitis, was present in 17 autopsied patients $(44 \%)$. MNGC were distributed in the brain in two pat- terns: scattered discrete MGN in white and grey matter surrounding one to several MNGCs, and non-focal, diffuse distribution of MNGCs regionally throughout white matter tracts, sometimes with angiocentric collections of many macrophages and MNGCs. In this study a few patients displayed both patterns in different regions of the brain. The differentiation of these two overlapping patterns of HIV encephalitis has been previously reported, ${ }^{14,22,23}$ but here evolution of HIV brain infection starting as microglial nodules at the site of HIV penetration of the brain capillary endothelium, and extending through the white matter in a diffuse destructive infiltrative pattern regionally, is suggested by the observation that patients with the MGN-associated pattern have, on average, a shorter history of profound immunosuppression. In this study, cases with microglial nodule-associated MNGC had a shorter mean duration of survival with the diagnosis of AIDS than did patients with a diffuse distribution of MNGCs and the appearance of degenerative changes within white matter. Regions of vacuolated white matter without MNGCs present could result from past HIV infection, or represent connecting tracts with Wallerian degeneration and subsequent secondary demyelination.

Primary central nervous system lymphoma was found in 14 of 46 patients, while systemic lymphoma was present or had been previously treated in another 8 patients, including one with probable secondary central nervous system involvement by lymphoma. Zidovudine treatment was not significantly associated with an increased incidence of systemic lymphoma or primary central nervous system lymphoma. The mean duration of AIDS diagnosis was longer in patients with CNS lymphoma, in both zidovudine-treated and untreated patients, than the average survival of untreated patients with no lymphoproliferative disorders at autopsy (14.5 months and 13.3 months respectively, compared to 8.3 months for the latter). These observations tend to support the conclusion of Pluda and coauthors ${ }^{29}$ that longer duration of survival of AIDS patients with antiretroviral therapy will be associated with an increased incidence of lymphoproliferative disorders, but indicates that untreated patients with similarly long duration of survival with the diagnosis of AIDS are equally likely to develop lymphoma as those with zidovudine treatment.

Interesting observations on the association of primary central nervous system lymphoma with infection of the brain parenchyma by HIV or other opportunistic organisms, and the predominance of periventricular spread of lymphoma also emerge from this study. Nine of eleven autopsied patients with primary central nervous system lymphoma (all of B-cell morphology) had HIV encephalitis (6 patients), toxoplasmosis (4 patients), or CMV encephalitis ( 3 patients). HIV-type MNGC were present within or at the periphery of lymphoma lesions in four of these patients. HIV has been shown to be preferentially localized in CNS lymphoma lesions of AIDS patients by immunohistochemical methods. ${ }^{30}$ The periventricular distribution of lymphoma lesions in a majority of the cases examined here corresponds to the usual distribution of CMV encephalitis, ${ }^{31.32}$ and occasionally disseminated Toroplasma. The frequent occurrence of frontal lobe white matter and basal ganglia lymphoma also parallels these sites as the most frequent locations of HIV encephalitis in this group of patients. Two patients who had been treated in the past ( 3 years and 4 months before death) for cerebral toxoplasmosis with improvement of symptoms, and no evidence of 
toxoplasmosis at autopsy, eventually died with primary central nervous system lymphoma. One individual dying with extensive Toxoplasma lesions displayed a single focus of infiltrative lymphoma within one lesion. To.roplasma lesions of AIDS patients have been observed to be associated with intracerebral lymphoma. ${ }^{15.29}$ Patients without central nervous system lymphoproliferative lesions showed other brain infections in a smaller percentage of patients than the group with lymphoproliferation (40\% compared to $80 \%$ ). The suggestion is therefore made that AIDS central nervous system lymphoma develops in parenchymal inflammation responding to infectious destruction of parenchymal cells, by HIV or another opportunistic infection eliciting an inflammatory response. Morgello and coworkers have previously suggested this interpretation of the relationship of infection, particularly CMV encephalitis, with the occurrence of CNS lymphoma in AIDS, a relationship not found in CNS lymphoma of other patients. ${ }^{33}$

This investigation documents that the AIDS-associated neuropathology of a mostly homosexual risk factor cohort in Vancouver, British Columbia, is qualitatively the same as found in other areas, although quantitatively, lymphoma may be more frequent in neurologically symptomatic individuals. More extensive examination of the hypotheses that lymphoma is associated with prior central nervous system infection, including HIV-I infection of parenchymal cells, and that HIV central nervous system infection proceeds regionally with initial stages represented by $\mathrm{HIV}$-associated MGN and later stages by diffuse HIV MNGC in an infiltrative, destructive pattern, with predilection for the basal ganglia, is suggested.

\section{ACKNOWLEDGEMENTS}

The authors gratefully acknowledge the assistance of Carol Appleton in preparation of illustrations, and Diana Lenard Secor in manuscript preparation. This work was supported in part by NIMH T32 Training Grant MH19200 and Contract \#NOI-Al-72631 (HVV).

\section{REFERENCES}

1. Anders KH, Guerra WF, Tomiyasu U, et al. The neuropathology of AIDS: UCLA experience and review. Am J Pathol 1986; 124: 537-558.

2. Rhodes RH. Histopathology of the central nervous system in the acquired immunodeficiency syndrome. Hum Pathol 1987; 18: 636-643.

3. Levy RM, Janssen RS, Bush TJ, et al. Neuroepidemiology of acquired immunodeficiency syndrome. J Acquir Immune Defic Syndr 1988: 1: $31-40$

4. Kure K, Llena JF, Lyman WD, et al. Human immunodeficiency virus- 1 infection of the nervous system. An autopsy study of 268 adult, pediatric and fetal brains. Hum Pathol 1991; 22: 700-710.

5. Petito CK, Cho E-S, Lemann W, et al. Neuropathology of acquired immunodeficiency syndrome (AIDS): an autopsy review. J Neuropathol Exp Neurol 1986; 45: 635-646.

6. de la Monte SM, Ho DD, Schooley RT, et al. Subacute encephalomyelitis of AIDS and its relation to HTLV-III infection. Neurology 1987; 37: 562-569.

7. de Girolami U, Smith TW, Hènin D, et al. Neuropathology of the acquired immunodeficiency syndrome. Arch Pathol Lab Med 1990; 114: 643-655.

8. Moskowitz LB, Hensley GT, Chan JC, et al. Neuropathology of accuired immune deficiency syndrome. Arch Pathol Lab Med $1984 ; 108: 867-872$.
9. Lantos PL, McLaughlin JE, Scholtz CL. et al. Neuropathology of the brain in HIV infection. Lancet 1989; 1: 309-311.

10. Esiri MM, Scaravilli F, Millard PR, et al. Neuropathology of HIV infection in haemophiliacs: comparative necropsy study. $\mathrm{Br} \mathrm{Med}$ J 1989; 299: 1312-1315.

11. Hènin D, Duyckaerts C. Chaunu MP, et al. Etude neuropathologique de 31 cas de syndrome d'immuno-dèpression acquise. Rev Neurol (Paris) 1987; 143: 631-642.

12. Gray F, Gherardi R. Keohane C, et al. Pathology of the central nervous system in 40 cases of acquired immune deficiency syndrome (AIDS). Neuropathol Appl Neurobiol 1988; 14: 365-380.

13. Lang W, Miklossy J. Deruaz JP, et al. Neuropathology of the acquired immune deficiency syndrome (AIDS): a report of 135 consecutive autopsy cases from Switzerland. Acta Neuropathol 1989; 77: 379-390.

14. Kleihues P, Leib SL, Strittmatter C, et al. HIV encephalopathy: incidence, definition, and pathogenesis. Acta Pathol Jpn 1991: 41: 197-205.

15. Giampalmo A, Pesce C, Ardoino S, et al. Neuropathological findings in an autopsy series of Italian subjects with AIDS. Clin Neuropathol 1989; 8: 120-125.

16. Budka $H$, Costanzi $G$, Cristina $S$, et al. Brain pathology induced by infection with the human immunodeficiency virus (HIV). Acta Neuropathol (Berlin) 1987; 75: 185-198.

17. Stavrou D, Mehraein P, Mellert W. et al. Evaluation of intracerebral lesions in patients with acquired immunodeficiency syndrome. Neuropathological findings and experimental data. Neuropathol Appl Neurobiol 1989: 15: 207-222.

18. Rosemberg S, Lopes MBS. Tsanaclis AM. Neuropathology of acquired immunodeficiency syndrome (AIDS). Analysis of 22 Brazilian cases. J Neurol Sci 1986: 76: 187-198.

19. Funata N, Maeda $Y$, Koike $M$, et al. Neuropathology of the central nervous system in acquired immune deficiency syndrome (AIDS) in Japan. Acta Pathol Jpn 1991: 41: 206-211.

20. Dickson DW, Belman AL, Park YD, et al. Central nervous system pathology in pediatric AIDS: an autopsy study. APMIS 1989; (Suppl 8): 40-57.

21. Koppel BS, Wormser GP, Tuchman AJ, et al. Central nervous system involvement in patients with acquired immune deficiency syndrome (AIDS). Acta Neurologica Scand 1985: 71: 337-353.

22. Budka $\mathrm{H}$. The definition of HIV-specific neuropathology. Acta Pathol Jpn 1991; 41: 182-191.

23. Budka $\mathrm{H}$. Neuropathology of human immunodeficiency virus infection. Brain Pathol 1991; 1: 163-175.

24. Centers for Disease Control. Revision of the CDC surveillance case definition for acquired immunodeficiency syndrome. MMWR 1987; 36 (Suppl IS): IS-9S

25. Anders $\mathrm{KH}$, Latta $\mathrm{H}$, Chang BS, et al. Lymphomatoid granulomatosis and malignant lymphoma of the central nervous system in the acquired immunodeficiency syndrome. Hum Pathol 1989; 20: 326-334.

26. Vinters HV, Anders $\mathrm{KH}$, Barach $\mathrm{P}$. Focal pontine leukoencephalopathy in immunosuppressed patients. Arch Pathol Lab Med 1987; 111: 192-196.

27. Wiley CA, Nelson JA. Role of human immunodeficiency virus and cytomegalovirus in AIDS encephalitis. Am J Pathol 1988: 133: 73-81.

28. Schmidbauer M, Budka H, Okeda R, et al. Multifocal vacuolar leucoencephalopathy: a distinct HIV-associated lesion of the brain. Neuropathol Appl Neurobiol 1990: 16: 437-443.

29. Pluda JM, Yarchoan R, Jaffe ES, et al. Development of nonHodgkin lymphoma in a cohort of patients with severe human immunodeficiency virus (HIV) infection on long-term antiretroviral therapy. Ann Intern Med 1990; 113: 276-282.

30. Cornford ME, Said JW, Vinters HV. Immunohistochemical localization of human immunodeficiency virus (HIV) in central ner- 
vous system lymphoproliferative lesions of patients with AIDS Mod Pathol 1991; 4: 232-238.

31. Vinters HV, Kwok MK, Ho HW, et al. Cytomegalovirus in the nervous system of patients with the acquired immune deficiency syndrome. Brain 1989; 112: 245-268.

32. Vinters HV, Anders KH. Opportunistic infections: Cytomegalovirus. In Netropathology of AIDS. Boca Raton: CRC Press, $1990 ; 29-45$.
33. Morgello S, Petito CK, Mouradian JA. Central nervous system lymphoma in the acquired immunodeficiency syndrome. Clin Neuropathol 1990; 9: 205-215. 